\title{
Correlation between magnetic resonance imaging and cone-beam computed tomography for maxillary sinus graft assessment
}

\author{
Fernando Antonio Reis Laurino $\mathbb{D}^{1}$, Isabela Goulart Gil Choi $\mathbb{D}^{1}$, Jun Ho Kim(D) ${ }^{1}$, Ivan Onone Gialain $\mathbb{D}^{1,2}$, \\ Renato Ferraço $\mathbb{D}^{3}$, Rainer Guilherme Haetinger $\mathbb{D}^{4}$, Otavio Henrique Pinhata-Baptista $\mathbb{D}^{3}$, \\ Reinaldo Abdala-Junior $\mathbb{D}^{1,5}$, Claudio Costa $\mathbb{D}^{1}$, Arthur Rodriguez Gonzalez Cortes $\mathbb{D}^{1,6, *}$ \\ ${ }^{1}$ Department of Stomatology, School of Dentistry, University of São Paulo, São Paulo, Brazil \\ ${ }^{2}$ Stricto Sensu Research Program on Integrated Dental Sciences, Universidade de Cuiabá, Cuiabá, Brazil \\ ${ }^{3}$ Department of Implantology, Military Hospital of São Paulo Area, São Paulo, Brazil \\ ${ }^{4}$ Department of Radiology, Hospital Beneficiência Portuguesa of São Paulo, São Paulo, Brazil \\ ${ }^{5}$ Department of Radiology, Centro Universitário Sudoeste Paulista, Avaré, São Paulo, Brazil \\ ${ }^{6}$ Department of Dental Surgery, Faculty of Dental Surgery, University of Malta, Msida, Malta
}

\section{ABSTRACT}

Purpose: Little is known regarding the accuracy of clinical magnetic resonance imaging (MRI) protocols with acceptable scan times in sinus graft assessment. The aim of this study was to evaluate the correlations between MRI and cone-beam computed tomographic (CBCT) measurements of maxillary sinus grafts using 2 different clinical MRI imaging protocols.

Materials and Methods: A total of 15 patients who underwent unilateral sinus lift surgery with biphasic calcium phosphate were included in this study. CBCT, T1-weighted MRI, and T2-weighted MRI scans were taken 6 months after sinus lift surgery. Linear measurements of the maximum height and buccolingual width in coronal images, as well as the maximum anteroposterior depth in sagittal images, were performed by 2 trained observers using CBCT and MRI Digital Imaging and Communication in Medicine files. Microcomputed tomography (micro-CT) was also performed to confirm the presence of bone tissue in the grafted area. Correlations between MRI and CBCT measurements were assessed with the Pearson test.

Results: Significant correlations between CBCT and MRI were found for sinus graft height (T1-weighted, $\mathrm{r}=0.711$ and $P<0.05$; T2-weighted, $\mathrm{r}=0.713$ and $P<0.05$ ), buccolingual width (T1-weighted, $\mathrm{r}=0.892$ and $P<0.05$; T2-weighted, $\mathrm{r}=0.956$ and $P<0.05$ ), and anteroposterior depth (T1-weighted, $\mathrm{r}=0.731$ and $P<0.05 ;$ T2-weighted, $\mathrm{r}=0.873$ and $P<0.05$ ). The presence of bone tissue in the grafted areas was confirmed via micro-CT.

Conclusion: Both MRI pulse sequences tested can be used for sinus graft measurements, as strong correlations with CBCT were found. However, correlations between T2-weighted MRI and CBCT were slightly higher than those between T1-weighted MRI and CBCT. (Imaging Sci Dent 2020; 50: 93-8)

KEY WORDS: Cone-Beam Computed Tomography; Magnetic Resonance Imaging; Alveolar Ridge Augmentation

\section{Introduction}

Low bone density and insufficient bone volume are

Received September 7, 2019; Revised January 22, 2020; Accepted February 13, 2020 *Correspondence to : Prof. Arthur Rodriguez Gonzalez Cortes

Department of Stomatology, School of Dentistry, University of São Paulo, Av. Professor Lineu Prestes 2227, Zip code: 05508-000, São Paulo, SP, Brazil Tel) 55-11-3091-7831,E-mail) arthuro@usp.br common conditions of edentulous posterior maxillae..$^{1-4}$ For this reason, dental implant placement in such cases may require an additional grafting procedure..$^{5-7}$ In this context, sinus floor grafting using the lateral window approach following virtual 3-dimensional (3D) planning is a predictable way to achieve sufficient bone volume for 
dental implant therapy. ${ }^{89}$

Preoperative virtual planning using cone-beam computed tomography (CBCT) with dedicated software has been considered useful to obtain predictable outcomes for posterior implant placement. ${ }^{10,11}$ However, despite studies demonstrating that the doses of ionizing radiation associated with CBCT are significantly lower than those associated with computed tomography, $\mathrm{X}$-ray doses still pose risks for patients and should be avoided or minimized as much as possible according to the "as low as reasonably achievable" (ALARA) radiation guidelines. ${ }^{12,13}$

While CBCT is the most common imaging method for surgical planning, magnetic resonance imaging (MRI) has been used in several fields of dentistry, offering enhanced soft tissue imaging resolution without exposing the patient to ionizing radiation. ${ }^{14-20}$ Despite recent evidence that MRI is useful for surgical planning and assessment of the alveolar bone, ${ }^{15,18,20}$ little is known regarding the accuracy of clinical MRI protocols in assessing sinus grafted areas for implant planning. ${ }^{14-21}$

Thus, the aim of this study was to correlate linear measurements of maxillary sinus grafts made with MRI and CBCT, using MRI protocols with pulse sequence parameters and scanning times acceptable for patients.

\section{Materials and Methods}

The present study was approved by the ethics committee of the University of São Paulo (Comitê de Ética em Pesquisa, protocol no. 82404018.0.0000.0075). All subjects signed an informed consent form. This investigation was conducted according to the principles of the Declaration of Helsinki.

\section{Inclusion and exclusion criteria}

This retrospective study was conducted with 15 patients who had undergone unilateral sinus floor augmentation. In all cases, an initial CBCT scan was taken for use in planning sinus lift surgery. In this scan, the presence of anatomical features such as sinus septa, the thickness of the lateral plate and the sinus membrane, and the buccolingual, anteroposterior, and inferosuperior sinus dimensions were assessed to plan the area to be grafted. The condition and $3 \mathrm{D}$ dimensions of the remaining native alveolar bone were also assessed. Then, 6 months after sinus lift surgery, all patients underwent T1- and T2-weighted MRI as well as another maxillary CBCT scan. Exclusion criteria included a history of bone metabolic diseases such as osteoporosis or of other grafting procedures.

\section{MRI acquisition}

All facial MRI scans were acquired with a 1.5-T MRI scanner (Aera; Siemens, Erlangen, Germany) using a head coil for the purpose of evaluating response to treatment. Two pulse sequences with fields of view of $199 \mathrm{~mm} \times 199$ $\mathrm{mm}$ and matrices of $448 \times 896$ were used: a spin-echo T1-weighted sequence with a coronal orientation (repetition time, $402 \mathrm{~ms}$; echo time, $10 \mathrm{~ms}$; number of averages, 2; bandwidth, 190; and slice thickness, $4 \mathrm{~mm}$ ) and a spinecho T2-weighted sequence with a coronal orientation (repetition time, $4100 \mathrm{~ms}$; echo time, $76 \mathrm{~ms}$; number of averages, 2; bandwidth, 260; and slice thickness, $4 \mathrm{~mm}$ ).

\section{CBCT acquisition}

CBCT scans (preoperative and 6 months after sinus lift surgery) of all patients were taken using the same CBCT device (Orthophos ${ }^{\circledR}$ XG3D; Sirona Dental Systems GmbH, Bensheim, Germany) with a specific protocol for dental implants (field of view, $8 \mathrm{~cm} \times 8 \mathrm{~cm}$ to include the full extension of both the maxillary sinuses and the maxillary dental arch; voxel size, $0.16 \mathrm{~mm}$, peak kilovoltage, $90 \mathrm{kVp}$, current, 3 to $8 \mathrm{~mA}$ ).

\section{Data analysis}

Digital Imaging and Communication in Medicine (DICOM) files from all CBCT and MRI scans were analyzed with imaging software (Osiri ${ }^{\circledR}$ version 3.9.4; Pixmeo, Geneva, Switzerland). Since this study was intended to address the clinical relevance and usefulness of MRI as an independent tool for measuring sinus grafted areas, anatomical landmarks were not used as references to ensure similarity between coronal MRI and CBCT slices. Instead, the coronal slices containing the greatest height and width of the sinus graft, as well as the sagittal slice containing the greatest depth of the sinus graft, were identified and used for all measurements for both CBCT and MRI scans. As such, all coronal CBCT images were paired with the corresponding coronal MRI slices (for instance, those containing the greatest graft height) from the same patient, which were selected during implant planning. Then, the maximum graft height (standardized as the distance between the lowest point of the sinus cortical floor and the highest graft point available in the selected DICOM images) and the maximum graft width (standardized as the distance between the most lingual graft point and the most buccal graft point available in the selected DICOM images) were measured for all coronal MRI and CBCT scans using the software's linear measurement tool. Similarly, the greatest anteroposterior depth of the sinus graft (standardized as the distance 
between the most anterior graft point and the most posterior graft point available in the selected DICOM images) was measured for paired sagittal MRI and CBCT images. All measurements were performed by 2 trained observers and recorded in millimeters. Two sets of observations were performed by each observer 2 weeks apart to avoid memory bias. All images were assessed in a quiet, dimly-lit room.

\section{Micro-computed tomographic analysis}

Fragments of bone grafts of 10 representative cases were retrieved from the implant site using a CBCT surgical guide less than 1 week after the imaging scans were taken. For this purpose, a 3-mm-diameter trephine was used to retrieve the 6-mm-long bone fragment at the time of implant placement using a trephine bur (3-mm internal diameter) to prepare the implant site. A 3D-printed surgical guide was used to ensure that the bone fragment would be retrieved from the same point at the alveolar crest and along the same angle established during virtual implant planning. The fragment remained in the trephine after implant installation and was scanned with micro-computed tomography (micro-CT) (SkyScan $1172^{\circledR}$; SkyScan, Kontich, Belgium). The parameters used were $100 \mathrm{kV}$ and $100 \mu \mathrm{A}$, a spatial

Table 1. Descriptive data showing mean linear measurements obtained with cone-beam computed tomography (CBCT) and magnetic resonance imaging (MRI), as well as mean morphometric measurements obtained via micro-computerized tomography

(unit: $\mathrm{mm}$ )

\begin{tabular}{lccc}
\hline Variables method & Height & Width & Depth \\
\hline CBCT & $1.68 \pm 0.24$ & $1.80 \pm 0.29$ & $2.34 \pm 0.41$ \\
T1-weighted MRI & $1.61 \pm 0.29$ & $1.73 \pm 0.32$ & $2.35 \pm 0.47$ \\
T2-weighted MRI & $1.62 \pm 0.30$ & $1.78 \pm 0.33$ & $2.24 \pm 0.43$ \\
\hline
\end{tabular}

resolution of $6.0 \mu \mathrm{m}$, and a $0.5-\mathrm{mm} \mathrm{Al}$ filter. The time exposure per frame was $450 \mathrm{~ms}$. X-ray images were reconstructed with Nrecon ${ }^{\circledR}$ software (Sky-Scan), after which they were analyzed for bone volume fraction (bone volume per total volume $[\mathrm{BV} / \mathrm{TV}])$.

\section{Statistical analysis}

The sample size required to detect a minimum correlation of 0.7 and a statistical power of $80 \%$ with a significance level of $5 \%$ was calculated. Normality was assessed using the Shapiro-Wilk test. The levels of intraobserver and interobserver agreement were calculated using the intraclass correlation coefficient (ICC). The mean differences between the CBCT and T1-weighted MRI measurements, as well as between the CBCT and T2-weighted MRI measurements, were assessed with the Student $t$-test. Correlations between MRI, CBCT, and micro-CT measurements were assessed using the Pearson test. All analyses were performed using SPSS Statistics 17 software (SPSS Inc., Chicago, IL, USA). A $P$-value less than 0.05 was considered to indicate statistical significance.

\section{Results}

Descriptive statistics are available in Table 1. A sample of 15 patients ( 10 women and 5 men; mean age, $58.93 \pm$ 7.47 years), totalling 30 sinus graft coronal images (15 MRI and 15 CBCT images, 1 of each per patient), was included in this study (Table 1). A normal distribution was confirmed for all continuous variables $(P>0.05)$. High levels of intraobserver and interobserver agreement were found for both MRI and CBCT (ICC ranging from 0.83 to 0.87 , $P<0.05)$. No significant mean differences between CBCT and T1-weighted MRI or between CBCT and T2-weight-
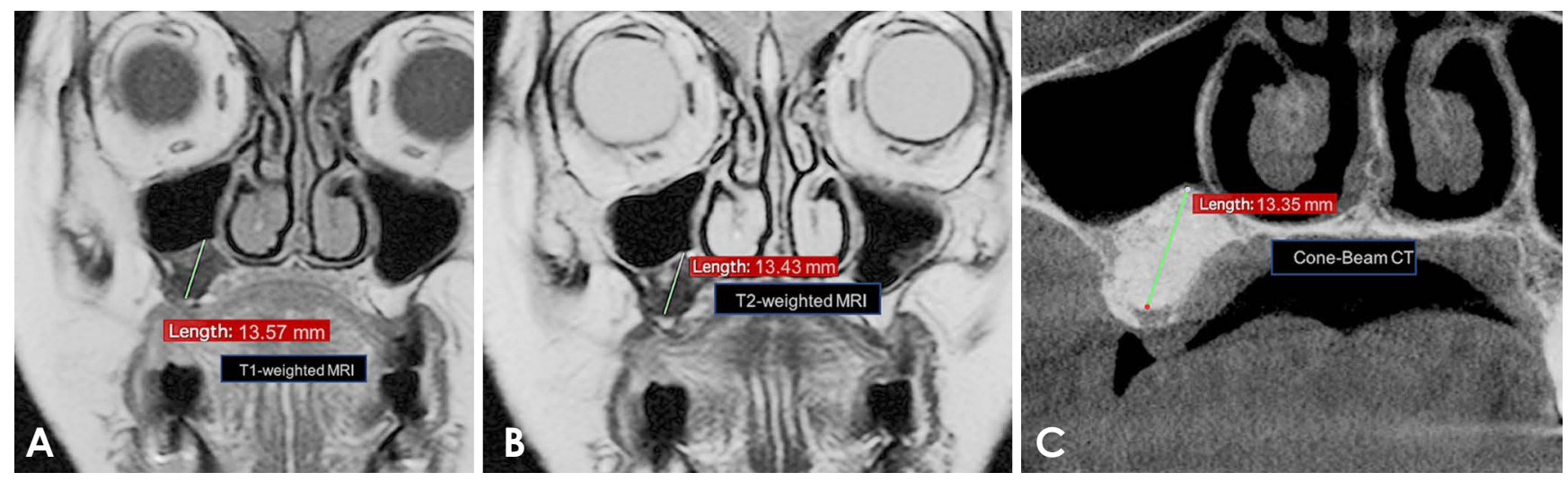

Fig. 1. Correlation between measurements made using magnetic resonance imaging (MRI) and cone-beam computed tomography (CBCT). In this case, the same measured value is obtained using all 3 methods. A. T1-weighted MRI. B. T2-weighted MRI. C. CBCT image. 

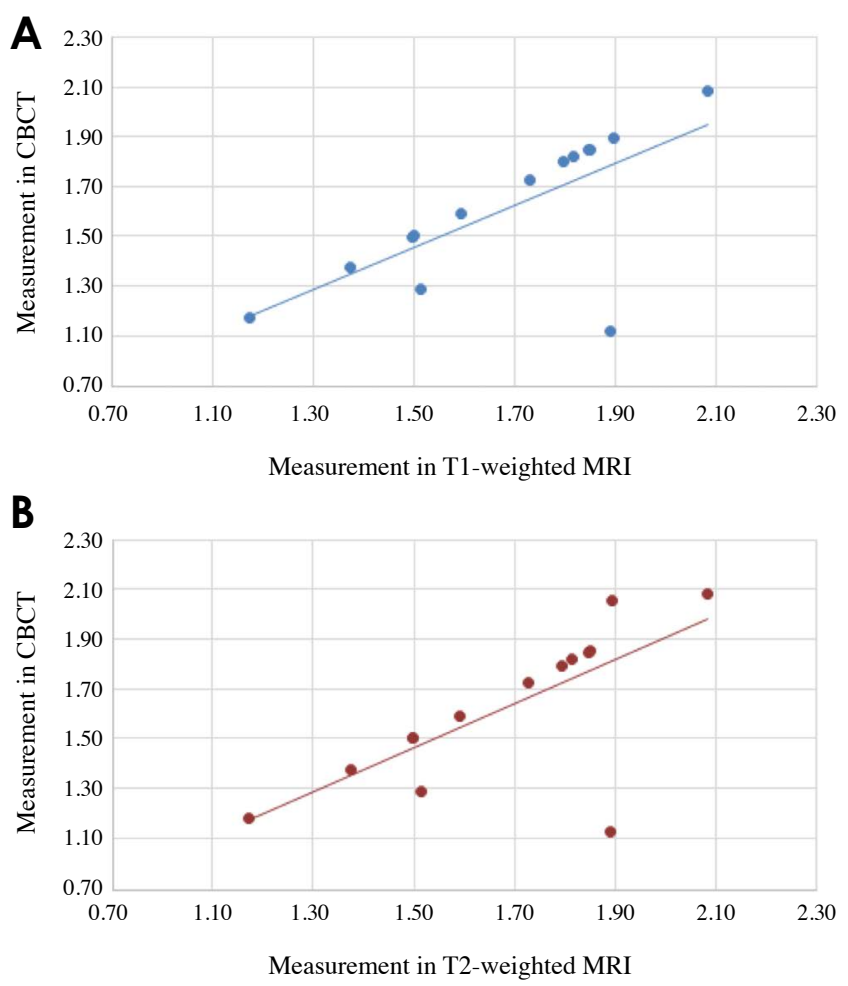

Fig. 2. Pearson correlation test result. A. Scatter dot graph shows a strong direct significant correlation between measurements made using cone-beam computed tomography (CBCT) and T1-weighted magnetic resonance image (MRI). B. Scatter dot graph shows a strong direct significant correlation between measurements made using CBCT and T2-weighted MRI.

ed MRI were found for the height, width, or depth measurements $(P>0.05)$. In addition, strong direct, significant correlations between CBCT and MRI were found for sinus graft height (T1-weighted, $\mathrm{r}=0.711$ and $P<0.05$; T2-weighted, $\mathrm{r}=0.713$ and $P=0.004$; Fig. 1), buccolingual width (T1-weighted, $\mathrm{r}=0.892$ and $P<0.05$; T2-weighted, $\mathrm{r}=0.956$ and $P<0.05$, Fig. 2), and anteroposterior depth (T1-weighted, $\mathrm{r}=0.731, P<0.05$; T2-weighted, $\mathrm{r}=0.873$ and $P<0.05)$. Furthermore, very strong correlations were found between T1- and T2-weighted MRI for graft height $(\mathrm{r}=0.981, P<0.05)$, buccolingual width $(\mathrm{r}=0.948$, $P<0.05)$, and anteroposterior depth $(\mathrm{r}=0.778, P<0.05)$. Micro-CT analyses revealed a high mean percentage of bone tissue (mean BV/TV, $65.44 \% \pm 8.71 \%$ ) present in the bone fragments (Figs. 3 and 4), indicating that all imaging measurements in this study were performed on areas with original and newly formed bone tissue. However, significant correlations were not found between micro-CT $\mathrm{BV} / \mathrm{TV}$ and MRI linear measurements $(P>0.05)$ or between micro-CT BV/TV and CBCT linear measurements $(P>0.05)$.

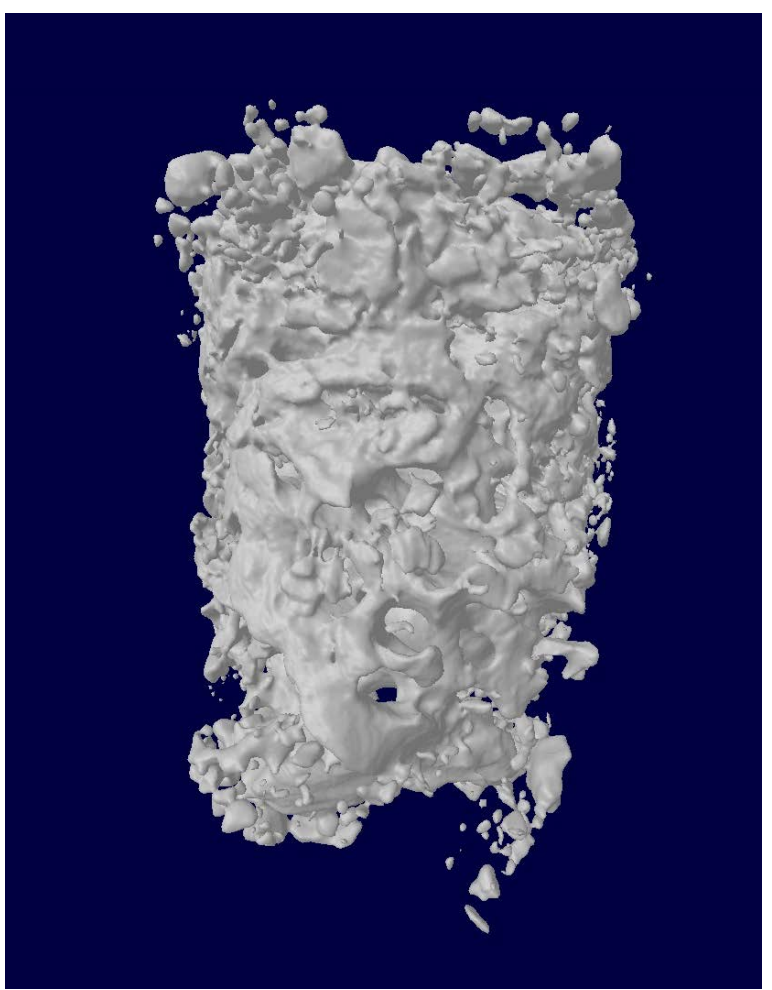

Fig. 3. Micro-computed tomographic 3-dimensional reconstruction image of a representative case, shows a satisfactory amount of mineralized bone tissue.

\section{Discussion}

Imaging and structural findings of alveolar bone have been correlated with bone grafts and dental implant outcomes $^{22,23}$ as well as with systemic bone findings. ${ }^{24}$ Along similar lines, prior to sinus lift procedures, diagnostic imaging is generally required for obtaining detailed information regarding the anatomy of the maxillary sinus for surgical planning, as well as for postoperative evaluation. In this context, CBCT has been commonly used, both to obtain linear measurements of the implant site and to assess pixel values proportional to the bone density of the sinus grafting areas. ${ }^{25}$ In contrast, non-ionizing MRI has also been suggested as a promising method to assess the maxillary sinuses for graft planning and follow-up. The goal of this study was to address the potential of MRI as an independent tool for performing maxillary sinus graft measurements. Thus, we assessed mean differences and correlations between CBCT and MRI images using 2 of the most common clinical MRI pulse sequences: spinecho T1- and T2-weighted imaging.

The present results support a previous study that also used both T1- and T2-weighted MRI for sinus lift assess- 


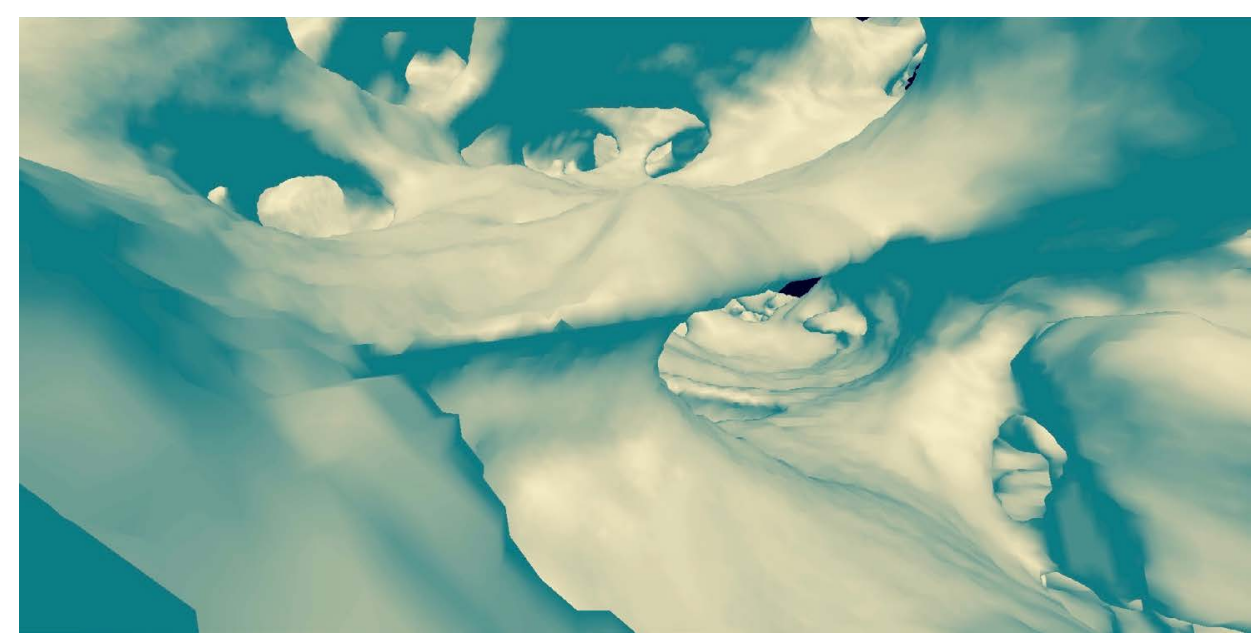

Fig. 4. Micro-computed tomographic 3-dimensional reconstruction of the same case as Figure 3, shows pores and trabecular bone tissue.

ment. ${ }^{18}$ However, that study lacked any comparison with the CBCT images commonly used today. Furthermore, different pulse sequences were used. Therefore, our study is the first to find strong correlations between CBCT and MRI using both T1- and T2-weighted pulse sequences with scan times acceptable for patients. In addition, we also found a very strong correlation between $\mathrm{T} 1$ - and $\mathrm{T} 2$-weighted images for maxillary sinus graft measurements. These results can be explained by the concentration of hydrogen atoms and related molecules within the healed graft, in which new bone formation had occurred. Although fat tissue from bone marrow appears hyperintense on T1-weighted imag$\mathrm{es},{ }^{20}$ fluid incorporation during sinus graft packing appears hyperintense on $\mathrm{T} 2$-weighted images. This explains why the correlations between T2-weighted MRI and CBCT presented herein were slightly stronger than those between T1-weighted MRI and CBCT.

In this study, only spin-echo MRI pulse sequences were used. In accordance with the observed strong correlation between MRI and CBCT, spin-echo sequences have been previously described to generally minimize MRI signal losses due to artefacts from dental materials relative to gradient-echo sequences. ${ }^{21}$ However, our favourable results contrast with other studies positing that spin-echo pulse sequences may lead to geometric distortion. ${ }^{26}$ The limitations of the present study include the low number of cases with micro-CT results, which could impact the statistical data involving morphometric micro-CT measurements. Similarly, other common MRI pulse sequences, such as gradient echo, could not be assessed in this study. Future prospective studies are recommended to address the influence of different pulse sequences and magnetic field strengths (e.g. using 3-T or 7-T MRI scanners) on the correlations presented herein.
In conclusion, within the limitations of this study, the present findings suggest that both clinical MRI T1- and T2-weighted pulse sequences can be used for sinus graft measurements, as strong correlations with CBCT measurements were found. However, the observed correlations between T2-weighted MRI and CBCT were slightly higher than those between T1-weighted MRI and CBCT.

\section{Conflicts of Interest: None}

\section{References}

1. Sekine H, Taguchi T, Seta S, Takano M, Takeda T, Kakizawa T. Dental implant treatment with different techniques for sinus floor elevation - a case report. Bull Tokyo Dent Coll 2007; 48: 87-91.

2. Lee KS, Kwon YH, Herr Y, Shin SI, Lee JY, Chung JH. Incomplete bone formation after sinus augmentation: a case report on radiological findings by computerized tomography at follow-up. J Periodontal Implant Sci 2010; 40: 283-8.

3. Cortes AR, Cortes DN, Arita ES. Effectiveness of piezoelectric surgery in preparing the lateral window for maxillary sinus augmentation in patients with sinus anatomical variations: a case series. Int J Oral Maxillofac Implants 2012; 27: 1211-5.

4. Tatum H Jr. Maxillary and sinus implant reconstructions. Dent Clin North Am 1986; 30: 207-29.

5. Chanavaz M. Maxillary sinus: anatomy, physiology, surgery, and bone grafting related to implantology - eleven years of surgical experience (1979-1990). J Oral Implantol 1990; 16: 199209.

6. Ulm CW, Solar P, Krennmair G, Matejka M, Watzek G. Incidence and suggested surgical management of septa in sinus-lift procedures. Int J Oral Maxillofac Implants 1995; 10: 462-5.

7. Khoury F. Augmentation of the sinus floor with mandibular bone block and simultaneous implantation: a 6-year clinical investigation. Int J Oral Maxillofac Implants 1999; 14: 55764.

8. Cortes AR, Ferraz P, Tosta M. Influence of etiologic factors in 
peri-implantitis: literature review and case report. J Oral Implantol 2012; 38: 633-7.

9. Tosta M, Cortes AR, Corrêa L, Pinto DdoS S Jr, Tumenas I, Katchburian E. Histologic and histomorphometric evaluation of a synthetic bone substitute for maxillary sinus grafting in humans. Clin Oral Implants Res 2013; 24: 866-70.

10. Wallace SS, Froum SJ. Effect of maxillary sinus augmentation on the survival of endosseous dental implants. A systematic review. Ann Periodontol 2003; 8: 328-43.

11. Del Fabbro M, Testori T, Francetti L, Weinstein R. Systematic review of survival rates for implants placed in the grafted maxillary sinus. Int J Periodontics Restorative Dent 2004; 24 : 565-77.

12. Spin-Neto R, Wenzel A. Patient movement and motion artefacts in cone beam computed tomography of the dentomaxillofacial region: a systematic literature review. Oral Surg Oral Med Oral Pathol Oral Radiol 2016; 121: 425-33.

13. Scarfe WC, Farman AG, Sukovic P. Clinical applications of cone-beam computed tomography in dental practice. J Can Dent Assoc 2006; 72: 75-80.

14. Mossa-Basha M, Blitz AM. Imaging of the paranasal sinuses. Semin Roentgenol 2013; 48: 14-34.

15. Aguiar MF, Marques AP, Carvalho AC, Cavalcanti MG. Accuracy of magnetic resonance imaging compared with computed tomography for implant planning. Clin Oral Implants Res 2008; 19: 362-5.

16. Bracher AK, Hofmann C, Bornstedt A, Hell E, Janke F, Ulrici J, et al. Ultrashort echo time (UTE) MRI for the assessment of caries lesions. Dentomaxillofac Radiol 2013; 42: 20120321.

17. Nagamatsu-Sakaguchi C, Maekawa K, Ono T, Yanagi Y, Minakuchi $\mathrm{H}$, Miyawaki S, et al. Test-retest reliability of MRIbased disk position diagnosis of the temporomandibular joint. Clin Oral Investig 2012; 16: 101-8.

18. Senel FC, Duran S, Icten O, Izbudak I, Cizmeci F. Assessment of the sinus lift operation by magnetic resonance imaging. $\mathrm{Br}$ J Oral Maxillofac Surg 2006; 44: 511-4.

19. Terra GT, Oliveira JX, Hernandez A, Lourenço SV, Arita ES, Cortes AR. Diffusion-weighted MRI for differentiation between sialadenitis and pleomorphic adenoma. Dentomaxillofac Radiol 2017; 46: 20160257.

20. Cortes AR, Cohen O, Zhao M, Aoki EM, Ribeiro RA, Abu Nada L, et al. Assessment of alveolar bone marrow fat content using 15 T MRI. Oral Surg Oral Med Oral Pathol Oral Radiol 2018; 125: 244-9.

21. Cortes AR, Abdala-Junior R, Weber M, Arita ES, Ackerman JL. Influence of pulse sequence parameters at $1.5 \mathrm{~T}$ and $3.0 \mathrm{~T}$ on MRI artefacts produced by metal-ceramic restorations. Dentomaxillofac Radiol 2015; 44: 20150136.

22. Cortes AR, Eimar H, Barbosa Jde S, Costa C, Arita ES, Tamimi F. Sensitivity and specificity of radiographic methods for predicting insertion torque of dental implants. J Periodontol 2015; 86: 646-55.

23. Cortes AR, Pinheiro LR, Cavalcanti MG, Arita ES, Tamimi F. Sinus floor bone failures in maxillary sinus floor augmentation: a case-control study. Clin Implant Dent Relat Res 2015; 17: 335-42.

24. Arita ES, Pippa MG, Marcucci M, Cardoso R, Cortes AR, Watanabe PC, et al. Assessment of osteoporotic alterations in achondroplastic patients: a case series. Clin Rheumatol 2013; 32: 399-402.

25. Nishimura DA, Aoki EM, Abdala Júnior R, Arita ES, Pinhata-Baptista $\mathrm{OH}$, Tateno RY, et al. Comparison of pixel values of maxillary sinus grafts and adjacent native bone with conebeam computed tomography. Implant Dent 2018; 27: 667-71.

26. Gray CF, Staff RT, Redpath TW, Needham G, Renny NM. Assessment of maxillary sinus volume for the sinus lift operation by three-dimensional magnetic resonance imaging. Dentomaxillofac Radiol 2000; 29: 154-8. 